\title{
On an Integral Arising in Mathematical Finance
}

Mark Craddock

\begin{abstract}
We consider an integral which arises in several problems in analysis and financial mathematics. We significantly simplify the integral, yielding a tractable form which is more useful for explicit calculations.
\end{abstract}

\section{Introduction}

The purpose of this paper is to present a tractable form for an integral that arises in a number of problems in analysis, financial mathematics and other areas. For example, it is connected to Asian option pricing, the Hartman-Watson law in stochastic calculus, as well as the problem of pricing zero coupon bonds in the Dothan model. (See below for the details). The integral in question was first derived by Yakubovich as a fundamental solution to the parabolic PDE

$$
u_{t}=x^{2} u_{x x}+x u_{x}-x^{2} u
$$

see [17]. The solution of this PDE can be obtained by using an index transform. This leads us to the so called Yakubovich heat kernel, (alternatively the Yakubovich integral $)^{1}$, which is defined by

$$
h_{t}(x, w)=\frac{2}{\pi^{2}} \int_{0}^{\infty} k e^{-k^{2} t} \sinh (\pi k) K_{i k}(x) K_{i k}(w) d k,
$$

where the function $K_{V}$ is McDonald's modified Bessel function, [1]. The Yakubovich integral does not seem to have been evaluated in closed form and the fact that we need to integrate over the index of two Bessel functions presents difficulties.

Mark Craddock

School of Mathematical Sciences, University of Technology Sydney, PO Box 123, Broadway, New

South Wales 2007, Australia. e-mail: Mark. Craddock@ut s . edu . au

${ }^{1}$ The integral derived by Yakubovich is slightly different, but equivalent to our formulation. 
The outline of the paper is as follows. First we provide motivation by showing how the PDE (1) arises when we seek to price an Asian option. Then we show that $h_{t}$ is actually the heat kernel for (1). Using the Fourier sine transform we are then able to show how the integral may be reduced to a simpler form, which may be explicitly evaluated as a series of error functions. Finally we discuss some financial applications.

\section{The Asian Option Pricing PDE}

The Yakubovich integral has a number of applications. To motivate our investigation, we consider Asian option pricing. Asian options are among the most popular of path dependent options currently traded. There is a very considerable literature on the subject and there are several alternative methods available for pricing. The first is to solve a pricing PDE. Much attention has been focused on the PDE introduced by Vecer [16]. Solving the PDE must be done numerically since no analytical solution satisfying the necessary boundary and terminal conditions is known. Secondly, one may attempt to numerically invert the Laplace transform of the price derived by Geman and Yor, [8], [2], [7], [6]. A fast and reasonably efficient third approach is to use the well known approximation for the Asian price derived by moment matching. Hull[11] describes this method. We can also use Monte Carlo simulation. The literature is extensive, but see [7] again. Finally we mention that a fast and accurate approximation using Taylor expansions was obtained by $\mathrm{Ju}$ [12].

Before Vecer introduced a simpler PDE, attention was focused on the PDE found in, for example, the introductory book by Dewynne, Howison and Wilmott [4]. This equation has two state variables, but is second order in only one of them. The problem of solving this PDE motivates the current work and leads directly to the Yakubovich heat kernel.

Suppose then that we are interested in pricing an Asian option with arithmetic average on the underlying $S=\{S(t): t \geq 0\}$, which follows geometric Brownian motion. That is

$$
d S(t)=r S(t) d t+\sigma S(t) d B(t), \quad S(0)=S_{0},
$$

with $B=\{B(t): t \geq 0\}$ a standard Brownian motion. Here $r$ denotes the short rate of interest and $\sigma$ is the volatility of the stock. Introduce $\xi(t)=\int_{0}^{t} S(\tau) d \tau$ and let $t \rightarrow T-t$. It is well known that if the price of the option is $V(S, \xi, t)=\bar{V}(\ln S, \xi, t)$ then

$$
\bar{V}_{t}=\frac{1}{2} \sigma^{2} \bar{V}_{y y}+\left(r-\frac{1}{2} \sigma^{2}\right) \bar{V}_{y}+e^{y} \bar{V}_{\xi}-r \bar{V}
$$

with $\bar{V}(\ln S, \xi, 0)=f(S, \xi)$ for some payoff $f$. Boundary conditions and the derivation of the PDE are in [4]. We now take the Laplace transform in $\xi$ and define 


$$
v(y, p, t)=\int_{0}^{\infty} \bar{V}(y, \xi, t) e^{-p \xi} d \xi .
$$

Integrating by parts gives

$$
\int_{0}^{\infty} e^{y} \bar{V}_{\xi}(y, \xi, t) e^{-p \xi} d \xi=p e^{y} v(y, p, t)-e^{y} \bar{V}(y, 0, t) .
$$

For the term $\bar{V}(y, 0, t)$ we observe that $\xi\left(t^{\prime}\right)=0$ implies that $S\left(t^{\prime}\right)=0$ for all $t<$ $t^{\prime}$. As an option on an underlying whose value is always zero would usually be worthless, it is reasonable to set $\bar{V}(y, 0, t)=\bar{V}(0,0, t)=0$. However if we do desire a nonzero value for $\bar{V}(y, 0, t)$, then this can be incorporated later using standard variation of parameter techniques. Our problem now is to solve the PDE

$$
v_{t}=\frac{1}{2} \sigma^{2} v_{y y}+\left(r-\frac{1}{2} \sigma^{2}\right) v_{y}+\left(p e^{y}-r\right) v,
$$

with $v(z, p, 0)=F\left(e^{z}, p\right)$ where $F$ denotes the Laplace transform of $f$ in the second variable. Equation (5) has time independent solutions in terms of Bessel functions. This motivates the change of variables

$$
v(z, p, t)=e^{\left(\frac{1}{2}-r / \sigma^{2}\right) z} e^{\frac{1}{8} \sigma^{2} \alpha^{2} t} U\left(\frac{2 \sqrt{2 e^{z} p}}{\sigma}, p, \frac{1}{8} \sigma^{2} t\right),
$$

where $\alpha=\left(2 r / \sigma^{2}+1\right)$ and $U$ satisfies

$$
U_{\tau}=y^{2} U_{y y}+y U_{y}+y^{2} U .
$$

This is very close to (1). Finally, letting $y=i x, u(y, p, \tau)=U(-i y, p, \tau)$, we have

$$
u_{\tau}=x^{2} u_{x x}+x u_{x}-x^{2} u
$$

subject to

$$
u(x, p, 0)=\left(\frac{i \sigma x}{\sqrt{8 p}}\right)^{2 r / \sigma^{2}-1} F\left(-\frac{\sigma^{2} x^{2}}{8 p}, p\right) .
$$

Thus the PDE (5) can be reduced to (1). A solution of this initial value problem is given by

$$
u(x, p, \tau)=\int_{0}^{\infty} u(w, p, 0) h_{\tau}(x, w) d w,
$$

where $h_{\tau}(x, w)$ is the heat kernel. So our next task is to compute this heat kernel. 


\section{The Yakubovich Heat Kernel}

We consider the PDE

$$
u_{t}=x^{2} u_{x x}+x u_{x}-x^{2} u
$$

with $u(x, 0)=\phi(x)$ for some bounded function $\phi$. Yakubovich has studied this problem in detail, see [17]. In [18] he relates the heat kernel for this equation to the Yor integral for the density arising from the Hartman-Watson law. In fact the PDE may be solved by means of the Kontorovich-Lebedev transform, which was introduced by M.I. Kontorovich and N.N. Lebedev in 1938, see [10] and [19]. We use the transform pair

$$
(\mathscr{K} g)(k)=\int_{0}^{\infty} \frac{1}{x} g(x) K_{i k}(x) d x,
$$

where $K_{i k}(x)$ is the modified Bessel function of the second kind, see [1]. The inversion integral is

$$
g(x)=\frac{2}{\pi^{2}} \int_{0}^{\infty} k \sinh (\pi k)(\mathscr{K} g)(k) K_{i k}(x) d k .
$$

Recall that the Bessel function $K_{i k}(x)$ satisfies the equation

$$
x^{2} K_{i k}^{\prime \prime}(x)+x K_{i k}^{\prime}(x)-\left(x^{2}-k^{2}\right) K_{i k}(x)=0 .
$$

We suppose that the solution of the PDE (7) is $u(x, t)$ and let

$$
\widehat{u}(k, t)=\int_{0}^{\infty} \frac{1}{x} u(x, t) K_{i k}(x) d x .
$$

Then assuming suitable behaviour at zero for $u$ we obtain

$$
\begin{aligned}
\widehat{u}_{t}(k, t) & =\int_{0}^{\infty} \frac{1}{x} u_{t}(x, t) K_{i k}(x) d x=\int_{0}^{\infty} \frac{1}{x}\left(x^{2} u_{x x}(x, t)+x u_{x}(x, t)-x^{2} u(x, t)\right) K_{i k}(x) d x \\
& \left.=\int_{0}^{\infty}\left(x u_{x x}(x, t)+u_{x}(x, t)-x u(x, t)\right)\right) K_{i k}(x) d x \\
& =\int_{0}^{\infty} u(x, t)\left(\left(x K_{i k}(x)\right)^{\prime \prime}-K_{i k}^{\prime}(x)-x K_{i k}(x)\right) d x \\
& =\int_{0}^{\infty} u(x, t)\left(x K_{i k}^{\prime \prime}(x)+K_{i k}^{\prime}(x)-x K_{i k}(x)\right) d x \\
& =\int_{0}^{\infty} \frac{1}{x} u(x, t)\left(x^{2} K_{i k}^{\prime \prime}(x)+x K_{i k}^{\prime}(x)-x^{2} K_{i k}(x)\right) d x \\
& =-k^{2} \int_{0}^{\infty} \frac{1}{x} u(x, t) K_{i k}(x) d x
\end{aligned}
$$


So the transformed solution $\widehat{u}$ satisfies $\widehat{u}_{t}(k, t)=-k^{2} \widehat{u}(k, t)$. Solving this gives $\widehat{u}(k, t)=\widehat{u}(k, 0) e^{-k^{2} t}$, where of course $\widehat{u}(k, 0)=(\mathscr{K} \phi)(k)$. Inverting $\widehat{u}(k, t)$ we have a solution to the initial value problem given by

$$
u(x, t)=\frac{2}{\pi^{2}} \int_{0}^{\infty}(\mathscr{K} \phi)(k) k \sinh (\pi k) K_{i k}(x) d k .
$$

This can be written as

$$
u(x, t)=\frac{2}{\pi^{2}} \int_{0}^{\infty} \int_{0}^{\infty} \phi(w) k e^{-k^{2} t} \sinh (\pi k) K_{i k}(x) K_{i k}(w) d k d w=\int_{0}^{\infty} \phi(w) h_{t}(x, w) d w
$$

where

$$
h_{t}(x, w)=\frac{2}{\pi^{2}} \int_{0}^{\infty} k e^{-k^{2} t} \sinh (\pi k) K_{i k}(x) K_{i k}(w) d k
$$

is the heat kernel for (7). We will refer to this as the Yakubovich heat kernel. We will prove the following result.

Theorem 3.1 The Yakubovich heat kernel is given by

$$
h_{t}(x, w)=\frac{1}{4 \sqrt{\pi} t^{3 / 2}} \int_{\cosh ^{-1}\left(\frac{x^{2}+w^{2}}{2 x w}\right)}^{\infty} \xi e^{-\frac{\xi^{2}}{4 t}} J_{0}\left(2 x w \cosh \xi-x^{2}-w^{2}\right) d \xi .
$$

Proof. The proof uses the Fourier sine transform define by

$$
\left(\mathscr{F}_{s} f\right)(y)=g(y)=\int_{0}^{\infty} f(k) \sin (k y) d k
$$

The inverse sine transform is

$$
\left(\mathscr{F}_{s}^{-1} g\right)(k)=f(k)=\frac{2}{\pi} \int_{0}^{\infty}\left(\mathscr{F}_{s} f\right)(y) \sin (k y) d k
$$

On page 189 of [14] we find the Fourier sine transform

$$
\begin{aligned}
\int_{0}^{\infty} \sinh (\pi x) K_{i x}(a) K_{i x}(b) \sin (x y) d x & =\mathscr{J}(u(a, b, y)) \\
& =\left\{\begin{array}{cc}
\frac{\pi^{2}}{4} J_{0}(u(a, b, y)), & u>0 \\
0, & u<0,
\end{array}\right.
\end{aligned}
$$

where $u(a, b, y)=2 a b \cosh (y)-a^{2}-b^{2}$. Here $J_{0}$ is the zeroth order Bessel function of the first kind [1]. Now we have by Fubini's Theorem 


$$
\begin{aligned}
h_{t}(x, w) & =\frac{2}{\pi^{2}} \int_{0}^{\infty} k e^{-k^{2} t} \mathscr{F}_{s}^{-1}(\mathscr{J}(u(a, b, \xi))(k) d k \\
& =\frac{4}{\pi^{3}} \int_{0}^{\infty} \int_{0}^{\infty} k e^{-k^{2} t} \sin (k y) \mathscr{J}(u(a, b, \xi) d k d \xi \\
& =\frac{2}{\pi^{2}} \int_{0}^{\infty} \frac{\xi e^{-\frac{\xi^{2}}{4 t}}}{2 \sqrt{\pi} t^{3 / 2}} \mathscr{J}(u(a, b, \xi)) d \xi \\
& =\frac{1}{4 \sqrt{\pi} t^{3 / 2}} \int_{\cosh ^{-1}\left(\frac{x^{2}+w^{2}}{2 x w}\right)}^{\infty} e^{-\frac{\xi^{2}}{4 t}} J_{0}\left(2 x w \cosh \xi-x^{2}-w^{2}\right) d \xi
\end{aligned}
$$

We finally note that for all positive $x$ and $w$, the quantity $\frac{x^{2}+w^{2}}{2 x w} \geq 1$, so that the lower bound of the integral is always real.

This is a form which is much easier to evaluate numerically than the original form, since we do not have to integrate with respect to the index of the Bessel function. The Integral can also be further reduced to the following result.

Corollary 3.1 The Yakubovich heat kernel $h_{t}(x, w)$ may be written

$$
\begin{aligned}
& h_{t}(x, w)=\frac{1}{\sqrt{4 \pi t}} \exp \left(-\frac{\left(\cosh ^{-1}\left(\frac{x^{2}+w^{2}}{2 x w}\right)\right)^{2}}{4 t}\right) \\
& +\frac{2 w x}{\sqrt{4 \pi t}} \int_{\cosh ^{-1}\left(\frac{x^{2}+w^{2}}{2 x w}\right)}^{\infty} \sinh \xi \exp \left(-\frac{\xi^{2}}{4 t}\right) J_{1}\left(x^{2}+w^{2}-2 x w \cosh \xi\right) d \xi .
\end{aligned}
$$

Equivalently

$$
\begin{aligned}
h_{t}(x, w) & =\frac{1}{\sqrt{4 \pi t}} \exp \left(-\frac{\left(\cosh ^{-1}\left(\frac{x^{2}+w^{2}}{2 x w}\right)\right)^{2}}{4 t}\right) \\
& +\frac{1}{\sqrt{4 \pi t}} \int_{0}^{\infty} \exp \left(-\frac{\left(\cosh ^{-1}\left(\frac{x^{2}+w^{2}+u}{2 x w}\right)\right)^{2}}{4 t}\right) J_{1}(u) d u .
\end{aligned}
$$

Proof. We simply integrate by parts and use the change of variable $u=w^{2}+x^{2}-$ $2 w x \cosh \xi$, and the relation $J_{0}^{\prime}(x)=-J_{1}(x)$, then replace $u$ with $-u$.

It is straightforward to expand the heat kernel in a series. From the definition of $J_{1}(x)([1], \mathrm{p} 360)$ one easily sees that

$$
J_{1}(u)=\frac{\left(x^{2}+w^{2}-2 x w \cosh \xi\right)}{2} \sum_{j=0}^{\infty} \frac{\left(x^{2}+w^{2}-2 x w \cosh \xi\right)^{2 j}}{2^{2 j} j !(j+1) !},
$$

with $u$ as in the preceding proof. The important point is that the integrals 


$$
\int_{\cosh ^{-1}\left(\frac{x^{2}+w^{2}}{2 x w}\right)}^{\infty} \sinh \xi \exp \left(-\frac{\xi^{2}}{4 t}\right) \cosh ^{l} \xi d \xi, \quad l=0,1,2,3, \ldots
$$

can all be evaluated in terms of the error function. Consequently we can expand the heat kernel as a series of error functions. For example

$$
\int_{\cosh ^{-1}\left(\frac{x^{2}+w^{2}}{2 w w}\right)}^{\infty} \sinh \xi e^{-\frac{\xi^{2}}{4 t}} d \xi=\frac{1}{2} \sqrt{\pi} e^{t} \sqrt{t}\left(\operatorname{erf}\left(\frac{a+2 t}{2 \sqrt{t}}\right)-\operatorname{erf}\left(\frac{a-2 t}{2 \sqrt{t}}\right)\right)
$$

where $a=\cosh ^{-1}\left(\frac{x^{2}+w^{2}}{2 x w}\right)$. So we have an expansion

$$
\begin{aligned}
h_{t}(x, w)= & \frac{1}{\sqrt{4 \pi t}} \exp \left(-\frac{\left(\cosh ^{-1}\left(\frac{x^{2}+w^{2}}{2 x w}\right)\right)^{2}}{4 t}\right) \\
& +\frac{w x}{4}\left(x^{2}+w^{2}\right) e^{t}\left(\operatorname{erf}\left(\frac{a+2 t}{2 \sqrt{t}}\right)-\operatorname{erf}\left(\frac{a-2 t}{2 \sqrt{t}}\right)\right) \\
& -\frac{1}{4} e^{4 t} w^{2} x^{2}\left(\operatorname{erfc}\left(\frac{a-4 t}{2 \sqrt{t}}\right)-4 \operatorname{erfc}\left(\frac{a+4 t}{2 \sqrt{t}}\right)\right)+\cdots
\end{aligned}
$$

Finally, since $\left|J_{1}(u)\right| \leq 3 / 5$ we have the easy estimate

$$
\begin{aligned}
\left|h_{t}(x, w)\right| \leq & \frac{1}{\sqrt{4 \pi t}} \exp \left(-\frac{\left(\cosh ^{-1}\left(\frac{x^{2}+w^{2}}{2 x w}\right)\right)^{2}}{4 t}\right) \\
& +\frac{3}{10 \sqrt{\pi t}} \int_{0}^{\infty} \exp \left(-\frac{\left(\cosh ^{-1}\left(\frac{x^{2}+w^{2}+u}{2 x w}\right)\right)^{2}}{4 t}\right) d u .
\end{aligned}
$$

We plot the kernel for $t=0.2$ in Figure 1 .

\section{Alternative Forms for $h_{t}(x, w)$.}

We may establish other equivalent forms for the heat kernel. One such is as follows.

\section{Proposition 4.1 Let}

$$
I=I(x, w, t)=\int_{-\infty}^{\infty} k e^{-k^{2} t} \sinh (\pi k) K_{i k}(x) K_{i k}(w) d k .
$$

Then 


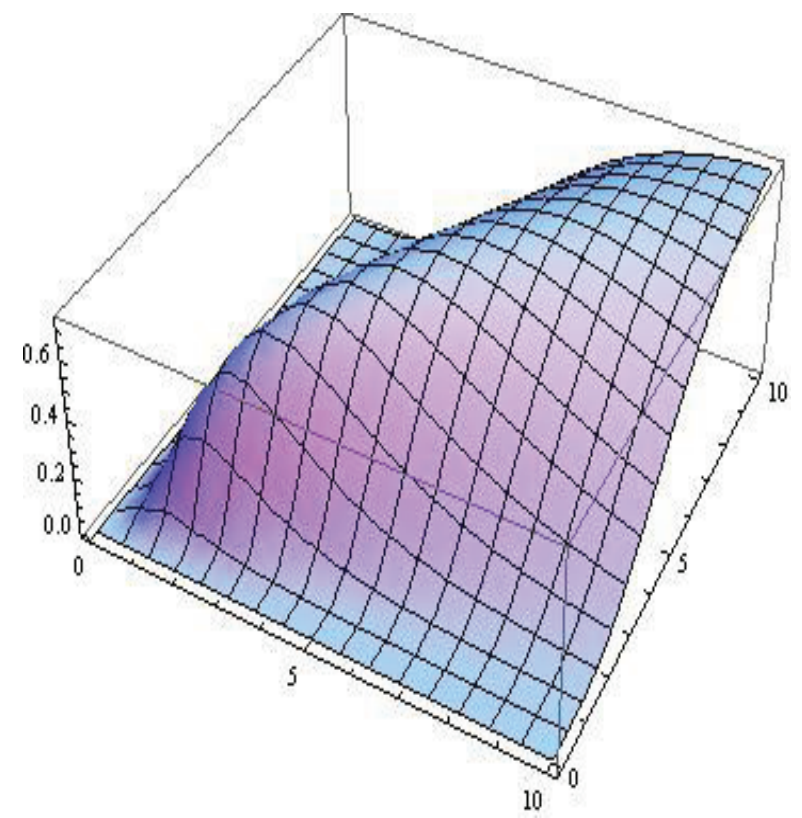

Fig. 1 The Yakubovich Heat Kernel at $t=0.2$

$$
I=\sqrt{\frac{\pi}{t}} e^{\frac{\pi^{2}}{4 t}} \int_{-\infty}^{\infty} e^{\frac{-\xi^{2}}{4 t}} \sin \left(\frac{\pi \xi}{2 t}\right) \sinh \xi \frac{K_{1}\left(\sqrt{x^{2}+w^{2}+2 x w \cosh \xi}\right)}{\sqrt{x^{2}+w^{2}+2 x w \cosh \xi}} d \xi
$$

and $h_{t}(x, w)=\frac{1}{2} I$.

Proof. The second part follows trivially from the fact that the integrand is even in $k$. Now we use the identities

$$
K_{i k}(u)=\int_{0}^{\infty} e^{-u \cosh \xi+i k \xi} d \xi
$$

and

$$
K_{i k}(x) K_{i k}(w)=\frac{1}{2} \int_{-\infty}^{\infty} \exp \left(-\frac{u w}{2 x}-\frac{x\left(u^{2}+w^{2}\right)}{2 u w}\right) K_{i k}(u) \frac{d u}{u},
$$

see [19]. Using Fubini's Theorem we have 


$$
\begin{aligned}
I(x, w, t)=\frac{1}{2} & \int_{-\infty}^{\infty} \int_{-\infty}^{\infty} \int_{0}^{\infty} k e^{-k^{2} t} \sinh (\pi k) \\
& \times \exp \left(-\frac{u w}{2 x}-\frac{x\left(u^{2}+w^{2}\right)}{2 u w}\right) e^{-u \cosh \xi+i k \xi} \frac{d u}{u} d k d \xi .
\end{aligned}
$$

Now from [9] we have the integral (which can also be done in Mathematica 9),

$$
\int_{0}^{\infty} e^{-\frac{u w}{2 x}-\frac{x\left(u^{2}+w^{2}\right)}{2 u w}} e^{-u \cosh \xi} \frac{d u}{u}=2 K_{0}\left(\sqrt{x^{2}+w^{2}+2 x w \cosh \xi}\right) .
$$

So that

$$
\begin{aligned}
I & =\int_{-\infty}^{\infty} \int_{-\infty}^{\infty} k e^{-k^{2} t} \sinh (\pi k) e^{i k \xi} K_{0}\left(\sqrt{x^{2}+w^{2}+2 x w \cosh \xi}\right) d k d \xi \\
& =-i \sqrt{\frac{\pi}{4 t}} \int_{-\infty}^{\infty} \frac{d}{d \xi}\left[e^{\frac{(\pi+i \xi)^{2}}{4 t}}-e^{\frac{(\pi-i \xi)^{2}}{4 t}}\right] K_{0}\left(\sqrt{x^{2}+w^{2}+2 x w \cosh \xi}\right) d \xi \\
& =\sqrt{\frac{\pi}{t}} e^{\frac{\pi^{2}}{4 t}} \int_{-\infty}^{\infty} e^{-\frac{\xi^{2}}{4 t}} \sin \left(\frac{\pi \xi}{2 t}\right) \sinh (\xi) \frac{K_{1}\left(\sqrt{x^{2}+w^{2}+2 x w \cosh \xi}\right)}{\sqrt{x^{2}+w^{2}+2 x w \cosh \xi}} d \xi
\end{aligned}
$$

upon integrating by parts and using Euler's formula to reduce the exponential terms. We made use of the identity $K_{0}^{\prime}(x)=-K_{1}(x)$.

Another approach to the integral is to observe that

$$
I(x, w, t)=\left.\frac{\partial}{\partial \alpha} \int_{-\infty}^{\infty} e^{-k^{2} t} \cosh (\alpha k) K_{i k}(x) K_{i k}(w) d k\right|_{\alpha=\pi} .
$$

By using the same arguments as in the previous proposition we have the following result.

Proposition 4.2 Let

$$
J(x, w, t)=\int_{-\infty}^{\infty} e^{-k^{2} t} \cosh (\alpha k) K_{i k}(x) K_{i k}(w) d k
$$

Then

$$
J(x, w, t)=2 \sqrt{\frac{\pi}{t}} e^{\frac{\alpha^{2}}{4 t}} \int_{-\infty}^{\infty} e^{-\frac{\xi^{2}}{4 t}} \cos \left(\frac{\alpha \xi}{2 t}\right) K_{0}\left(\sqrt{x^{2}+w^{2}+2 x w \cosh \xi}\right) d \xi .
$$

Since $K_{0}(x)$ decays exponentially it should be possible to numerically evaluate these integrals quite efficiently. 


\section{Some Applications}

For brevity we will only sketch the applications. The forthcoming paper [3] will address these in considerable detail.

\subsubsection{Asian Options}

The initial motivation for this investigation was the problem of pricing an Asian option. Our results on the Yakubovich heat kernel immediately give us the solution of the reduced form of the pricing equation for an Asian option derived previously. We have

$$
\begin{aligned}
& u(x, p, \tau)=\frac{1}{\sqrt{4 \pi \tau}} \int_{0}^{\infty} u(x, p, 0) \exp \left(-\frac{\left(\cosh ^{-1}\left(\frac{x^{2}+w^{2}}{2 x w}\right)\right)^{2}}{4 \tau}\right) d w \\
& +\frac{1}{\sqrt{4 \pi \tau}} \int_{0}^{\infty} \int_{0}^{\infty} u(x, p, 0) \exp \left(-\frac{\left(\cosh ^{-1}\left(\frac{x^{2}+w^{2}+u}{2 x w}\right)\right)^{2}}{4 \tau}\right) J_{1}(u) d u d w
\end{aligned}
$$

where $u(x, p, 0)=\left(\frac{i \sigma w}{\sqrt{8 p}}\right)^{2 r / \sigma^{2}-1} F\left(-\frac{\sigma^{2} w^{2}}{8 p}, p\right)$. Back substitution will lead us to the Laplace transform in the $\xi$ variable for the Asian option and the result depends on the payoff $f$ that we pick. One interesting question is how this relates to the Geman-Yor formula? One can relate the Geman-Yor formula to our result, but the details are involved, so we defer them to [3]. We note that there are already efficient methods for pricing Asian options, but this method does have some attractive features. Most notably, the double integral term can often be ignored. This requires justification, but similar comments hold for the Dothan bond pricing model below, and as this requires less prepatory material, we will present some preliminary numerical examples below for this model.

\subsubsection{Yor's Integral}

We note that there is another connection between Asian option pricing and the Yakubovich integral. In 1980 Yor expressed a density which is related to the Hartman-Watson law as an integral involving only elementary functions, [20]. Specifically, the density defined by the Laplace transform

$$
\int_{0}^{\infty} e^{-\lambda t} d \eta_{r}(t)=\frac{I_{\sqrt{2 \lambda}}(r)}{I_{0}(r)}
$$

has the density 


$$
p(t, r)=\frac{1}{\sqrt{2 \pi t^{3}} I_{0}(r)} H_{t}(r)
$$

where

$$
H_{t}(r)=\frac{r t}{\pi} \int_{0}^{\infty} \exp \left(\frac{1}{2 t}\left(\pi^{2}-x^{2}\right)-r \cosh x\right) \sinh x \sin \left(\frac{\pi x}{t}\right) d x
$$

The Hartman-Watson law is fundamental in deriving the Geman-Yor Laplace transform for the price of an Asian option, [8]. There is a direct relationship between the Yakubovich heat kernel and (18). In [18], Yakubovich showed that

$$
h_{t / 2}(x, w)=\int_{0}^{\infty} x \exp \left(-\frac{1}{2}\left(r \frac{x^{2}+w^{2}}{x w}+\frac{x w}{r}\right)\right) H_{t}(r) d r .
$$

As a consequence we have the following easy result.

Corollary 5.1 Let $r=\{r(t): t \geq 0\}$ be the process with density $p(t, r)$ defined by (16), with $r(t)=y$. Then

$$
\begin{aligned}
E_{x}\left[I_{0}((r(t))) \exp \left(-\frac{1}{2}\left(r(t) \frac{x^{2}+w^{2}}{x w}+\frac{x w}{r(t)}\right)\right)\right] \\
=\int_{0}^{\infty} I_{0}(r) \exp \left(-\frac{1}{2}\left(r \frac{x^{2}+w^{2}}{x w}+\frac{x w}{r}\right)\right) p(t, r) d r \\
=\frac{1}{\sqrt{2 \pi t^{3}} x}\left(\frac{1}{\sqrt{4 \pi t}} \exp \left(-\frac{\left.\left(\cosh ^{-1}\left(\frac{x^{2}+w^{2}}{2 x w}\right)\right)^{2}\right)}{4 t}\right)\right) \\
\left.+\frac{1}{\sqrt{4 \pi t}} \int_{0}^{\infty} \exp \left(-\frac{\left(\cosh ^{-1}\left(\frac{x^{2}+w^{2}+u}{2 x w}\right)\right)^{2}}{4 t}\right) J_{1}(u) d u\right)
\end{aligned}
$$

Here $E_{x}[f(r(t))]=E\left[f\left(r_{t}\right) \mid r(0)=x\right]$.

\subsubsection{Bond Pricing in the Dothan Model}

The Dothan model, [5], for the short rate of interest $r=\{r(t): t \geq 0\}$ is

$$
d r(t)=\frac{1}{2}(1-p) \sigma^{2} r(t) d t+\sigma r(t) d B(t)
$$

where $p \sigma / 2$ is the market price of risk. As usual, $\sigma$ denotes volatility and $B=\{B(t)$ : $t \geq 0\}$ is a standard Brownian motion. In [15], Pintoux and Privault prove that in the Dothan model, the price of a zero coupon bond $P(t, T)$ is given by $P(t, T)=$ $F\left(T-t, r_{t}\right)$, where 


$$
F(\tau, r)=\frac{2 p(2 r)^{p / 2}}{\sigma^{p}} \exp \left(-\frac{\sigma^{2} p^{2} \tau}{8}\right) \int_{0}^{\infty} e^{-p y} h_{\sigma^{2} \tau / 8}\left(\frac{\sqrt{8 r}}{\sigma}, e^{y}\right) d y
$$

Using our expression for the Yakubovich integral, we can express the bond price as

$$
\begin{aligned}
& F(\tau, r)=\alpha_{p, r}\left[\int_{0}^{\infty} \frac{e^{-p y}}{\sqrt{\pi \sigma^{2} \tau / 2}} \exp \left(-\frac{\cosh ^{-1}\left(\frac{\sigma\left(e^{2 y}+8 r / \sigma^{2}\right)}{2 e^{y} \sqrt{8 r}}\right)^{2}}{\sigma^{2} \tau / 2}\right) d y\right. \\
& \left.\quad+\int_{0}^{\infty} \int_{0}^{\infty} \frac{e^{-p y}}{\sqrt{\pi \sigma^{2} \tau / 2}} \exp \left(-\frac{\cosh ^{-1}\left(\frac{\sigma\left(e^{2 y}+8 r / \sigma^{2}+u\right)}{2 e^{y} \sqrt{8 r}}\right)^{2}}{\sigma^{2} \tau / 2}\right) J_{1}(u) d u d y\right]
\end{aligned}
$$

where $\alpha_{p, r}=\frac{2 p(2 r)^{p / 2}}{\sigma^{p}} e^{-\frac{1}{8} \sigma^{2} p^{2} \tau}$

\subsection{Numerical Implementation}

We will not attempt to establish the most efficient method of approximation, numerical evaluation of the various quantities derived above. Nor will we derive error estimates here. In [3] we will investigate these issues in detail. However, we will make a few preliminary observations about implementation and the consequences for pricing.

On the interval $[0,2 n]$ the Bessel function $J_{k}(u)$ is approximated by

$$
J_{k}(x) \approx \sum_{m=k}^{n} d_{k n m} x^{2 m-k}
$$

where

$$
d_{k n m}=\frac{(-1)^{m+k} 2^{k-2 m} n^{1-2 m}(n+m-1) !}{(m-k) !(n-m) !(m !)}
$$

see [13]. Thus

$$
\begin{aligned}
& h_{t}(x, w)=\frac{1}{4 \sqrt{\pi} t^{3 / 2}} \int_{\cosh ^{-1}\left(\frac{x^{2}+w^{2}}{2 x w}\right)}^{\infty} \xi e^{-\frac{\xi^{2}}{4 t}} J_{0}\left(2 x w \cosh \xi-x^{2}-w^{2}\right) d \xi \\
& \approx \frac{1}{4 \sqrt{\pi} t^{3 / 2}} \sum_{m=0}^{2 n} d_{0 n m} \int_{\cosh ^{-1}\left(\frac{x^{2}+w^{2}}{2 x w}\right)}^{2 n} \xi e^{-\frac{\xi^{2}}{4 t}}\left(2 x w \cosh \xi-x^{2}-w^{2}\right)^{2 m} d \xi
\end{aligned}
$$

The order of the polynomial approximation required will depend on the lower limit of integration. Each term in (23) can be evaluated in terms of exponential and error functions. Using this we may readily compute the value of the heat kernel and 
establish the prices in the models we are interested in. In practice we often need few terms of the series and in fact it is frequently the case that the first term will suffice, particularly for $t<1$. The in-built numerical routines in software such as Mathematica and Matlab can also be used to evaluate the integral with relative ease and high accuracy.

Actually, for the Dothan model, a good approximation can often be obtained simply by using the first term in (20), since the double integral is often small enough that we may neglect it. For example, consider the case when $r=0.02, \sigma=0.4$ and $p=2$, with time to maturity of two years. Evaluation of (19) using (20) gives a bond price of 0.291 . The contribution of the second integral is 0.006 . In fact for bonds with maturities less than two years, one can show numerically that the double integral makes essentially no contribution to the price. For example, with the same parameters as before and time to maturity of half a year, the bond price is 0.389 and the double integral only contributes 0.0004 . For longer maturities, we have the same effect. A ten year bond with the same parameters has a price of 0.182 , with the double integral contributing 0.0002 to the value.

A detailed investigation will be given in [3], but in practice

$$
F(\tau, r) \approx \alpha_{p, r} \int_{0}^{\infty} \frac{e^{-p y}}{\sqrt{\pi \sigma^{2} \tau / 2}} \exp \left(-\frac{\cosh ^{-1}\left(\frac{\sigma\left(e^{2 y}+8 r / \sigma^{2}\right)}{2 e^{y} \sqrt{8 r}}\right)^{2}}{\sigma^{2} \tau / 2}\right) d y
$$

will usually give an excellent approximation to the bond price. Similar comments hold for Asian options, but the discussion is rather more involved and so we defer it to [3].

\section{References}

1. Abramowitz, M., Stegun, I.: Handbook of Mathematical Functions, with Formulas, Graphs and Mathematical Tables, 10th edition. Dover, New York (1972).

2. Craddock, M., Heath, D., Platen, E.: Numerical Inversion of Laplace Transforms: A Survey of Techniques with Applications to Derivative Pricing. J. Computational Finance 4, 57-81 (2000).

3. Craddock, M., D. Roberts: Yakubovichs Integral and the Pricing of Derivative Securities. In Preparation, (2014).

4. Dewynne, J., Howison, S., Wilmott, P.: The Mathematics of Financial Derivatives. A Student Introduction. Cambridge University Press, Cambridge (1995).

5. Dothan, L.: On the term structure of interest rates. Jour. of Fin. Ec. 6, 59-69 (1978).

6. Eydeland, A., Geman, H.: Asian Options Revisited: Inverting the Laplace Transform. Risk 8, 65-67 (1995).

7. Fu, M., Madan, D., Wang, T.: Pricing Asian Options: A Comparison of Analytical and Monte Carlo Methods. University of Maryland, College of Business and Management, Working Paper (1995).

8. Geman, H., Yor, M.: Bessel Processes, Asian Options and Perpetuities. Mathematical Finance 3, 471-474 (1992). 
9. Gradshteyn, I., Ryzhik, I.: Table of Integrals, Series and Products. 6 edn, Academic Press (2000).

10. Gutiérrez-Tovar, Y., Méndez-Pérez, J.: The Kontorovich-Lebedev integral transformationwith a Hankel function kernel in a space of generalized functions of doubly exponential descent. J. Math. Anal. Appl. 328, 359-369 (2007).

11. Hull, J.C.: Options, Futures and Other Derivatives. Prentice Hall International, Inc., 3rd edition, (1997).

12. Ju, N.: Pricing Asian and basket options via Taylor expansion. J. Computational Finance 5, 79-103 (2002).

13. Millane, R. P., Eads, J. L.: Polynomial Approximations to Bessel Functions. IEEE Trans. Antennas and Propagation 51, 1398-1400 (2003).

14. Oberhettinger, F.: Tables of Fourier Transforms and Fourier Transforms of Distributions. Springer-Verlag, Berlin (1990).

15. Pintoux, C., Privault, N.: The Dothan pricing model revisited. Mathematical Finance 21, 355 363 (2011).

16. Vecer, J.: A new PDE approach for pricing arithmetic average Asian options. J. Computation Finance 4, 105-113 (2001).

17. Yakubovich, S.: The heat kernel and Heisenberg inequalities related to the KontorovichLebedev transform. Commun. Pure Appl. Anal 10, 745-760 (2011).

18. Yakubovich, S.: On the Yor integral and a system of polynomials related to the KontorovichLebedev transform. arXiv:1210.7309v1 (2012).

19. Yakubovich, S., Luchko, Y.: The Hypergeometric Approach to Integral Transforms and Convolutions. Mathematics And Its Applications 287 Kluwer, Dordrecht (1994).

20. Yor, M.: Loi de lindice du lacet Brownien et distribution de Hartman-Watson. Z. Wahrscheinlichkeitsth 53, 71-95 (1980). 